International Journal of Pure and Applied Mathematics

Volume 109 No. 2 2016, 235-243

ISSN: 1311-8080 (printed version); ISSN: 1314-3395 (on-line version)

url: http://www.ijpam.eu

doi: 10.12732/ijpam.v109i2.6

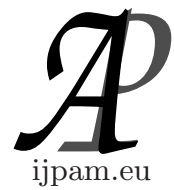

\title{
THE ORBIT GRAPH OF METACYCLIC \\ 2-GROUPS OF NEGATIVE TYPE
}

\author{
Mustafa Anis El-Sanfaz ${ }^{1}$, Nor Haniza Sarmin ${ }^{2}$, Sanaa Mohamed Saleh Omer ${ }^{3}$ \\ ${ }^{1,3}$ Department of Mathematics \\ Faculty of Science \\ University of Benghazi \\ Benghazi, LIBYA \\ ${ }^{1,2}$ Department of Mathematical Sciences \\ Faculty of Science \\ Universiti Teknologi Malaysia \\ Johor Bahru, MALAYSIA
}

\begin{abstract}
In this paper, let $G$ be a metacyclic 2-group of negative type of class two and class at least three. Let $\Omega$ be the set of all subsets of all commuting elements of size two in the form of $(a, b)$, where $a$ and $b$ commute and $|a|=|b|=2$. The orbit graph is a graph whose vertices are non-central orbits under group action of $G$ on $\Omega$. In this paper, the orbit graph of metacyclic 2-groups of negative type of nilpotency class two and class at least three is determined. Some graph properties are also provided.
\end{abstract}

AMS Subject Classification: 20P05, 20B40, 97K30

Key Words: commutativity degree, graph theory, orbit graph, group action

\section{Introduction}

Graph theory is the study of points and lines. More precisely, it involves the ways in which sets of vertices can be linked by edges. The concept in graph

Received: August 18, 2015

Published: September 8, 2016

${ }^{\S}$ Correspondence author (c) 2016 Academic Publications, Ltd.

url: www.acadpubl.eu 
theory is widely used among many fields and one of these uses are in group theory. In this section, some basic definitions that are needed in this paper are stated, starting with some definitions related to graph theory that can be found in one of the references (see [1] and [2]). A graph $\Gamma$ is a mathematical structure consisting of two sets namely vertices and edges which are denoted by $V(\Gamma)$ and $E(\Gamma)$, respectively. A graph is called directed if its edges are identified with ordered pair of vertices. Otherwise, $\Gamma$ is called indirected. Two vertices are connected if they are joined by an edge. A connected graph is a graph in which there is a partition of vertex $V$ into non empty subsets, $V_{1}, V_{2}, \ldots, V_{n}$ such that two vertices $x_{1}$ and $x_{2}$ are connected if and only if they belong to the same set $V_{i}$. Subgraphs $\Gamma\left(V_{1}\right), \Gamma\left(V_{2}\right), \ldots, \Gamma\left(V_{n}\right)$ are all components of $\Gamma$. The graph $\Gamma$ is connected, if it has precisely one component.

However, the graph is called complete if all of its vertices are adjacent to each other. In this paper, we denote the complete graph by $K_{n}$, where $n$ is the number of adjacent vertices. The graph is empty if there is no edge among its vertices and it is denoted by $K_{e}$. In addition, The graph is null if it has no vertices and it is denoted by $K_{0}$.

The following are some graph properties that are used in this paper.

A non-empty set $X$ of $\mathrm{V}(\Gamma)$ is called an independent set of $\Gamma$ if there is no adjacent between two elements of $X$ in $\Gamma$. However, the number of vertices in maximum independent set is called the independence number and is denoted by $\alpha(\Gamma)$. In addition, chromatic number is the minimum number $c$ for which $\Gamma$ is $c$-vertex colorable and is denoted by $\chi(\Gamma)$. The maximum number of complete subgraph is called the clique number and is denoted by $\omega(\Gamma)$. The diameter is the maximum distance between any two vertices of $\Gamma$ and $d(\Gamma)$ is used as its notation.

In 2013, Omer et al. [3] introduced a new graph called the orbit graph whose vertices are non-central orbits under group action on a set.

Since the metacyclic 2-groups of negative type are in the scope of this paper, thus the classifications of metacyclic 2-groups are stated in the following. In 2005, Beuerle [4] classified the metacyclic $p$-groups of nilpotency class two into two families of non-isomorphic 2-groups stated as follows:

(1) $G \cong\left\langle a, b: a^{2^{\alpha}}=b^{2^{\beta}}=1,[a, b]=a^{2^{\alpha-\gamma}}\right\rangle$, where $\alpha, \beta, \gamma \in \mathbb{N}, \alpha \geq 2 \gamma$ and $\beta \geq \gamma \geq 1$,

(2) $G \cong\left\langle a, b: a^{4}=1, b^{2}=[b, a]=a^{-2}\right\rangle$, namely the quaternion group of order 8, $Q_{8}$.

In addition, the metacyclic 2-groups of negative type of class at least three 
are partitioned into eight families [4]. The followings are some of the negative types which are considered in the scope of this research:

(3) $G \cong\left\langle a, b: a^{2^{\alpha}}=1, b^{2}=a^{2^{\alpha-1}},[b, a]=a^{-2}\right\rangle$, where $\alpha \geq 3$,

(4) $G \cong\left\langle a, b: a^{2^{\alpha}}=b^{2}=1,[b, a]=a^{-2}\right\rangle$, where $\alpha \geq 3$,

(5) $G \cong\left\langle a, b: a^{2^{\alpha}}=b^{2}=1,[b, a]=a^{2^{\alpha-1}-2}\right\rangle$, where $\alpha \geq 3$,

(6) $G \cong\left\langle a, b: a^{2^{\alpha}}=b^{2^{\beta}}=1,[b, a]=a^{-2}\right\rangle$, where $\alpha \geq 3, \beta>1$,

(7) $G \cong\left\langle a, b: a^{2^{\alpha}}=b^{2^{\beta}}=1,[b, a]=a^{2^{\alpha-1}-2}\right\rangle$, where $\alpha \geq 3, \beta>1$,

(8) $G \cong\left\langle a, b: a^{2^{\alpha}}=1, b^{2^{\beta}}=a^{2^{\alpha-1}},[b, a]=a^{2^{\alpha-\gamma}-2}\right\rangle$, where $\alpha-\gamma>1, \beta>$ $\gamma>1$

(9) $G \cong\left\langle a, b: a^{2^{\alpha}}=b^{2^{\beta}}=1,[b, a]=a^{2^{\alpha-\gamma}-2}\right\rangle$, where $\alpha-\gamma>1, \beta \geq \gamma>1$,

(10) $G \cong\left\langle a, b: a^{2^{\alpha}}=1, b^{2^{\beta}}=a^{2^{\alpha-1}},[b, a]=a^{-2}\right\rangle$, where $\alpha \geq 3, \beta>1$.

This paper is divided into three sections. The first section focuses on some background on graph theory and group theory, while the second section provides some recent publications related to the probability that a group element fixes a set and orbit graph. In the third section, we present our results.

\section{Preliminaries}

This section provides some previous works on orbit graph and the probability that a group element fixes a set.

The orbit graph was initially introduced by Omer et al. [3]. The vertices of this graph are non-central orbits under group action on a set. The following is the definition of orbit graph.

Definition 1. [3] Let $G$ be a finite group and $\Omega$ be a set of elements of $G$. Let $A$ be the set of commuting elements in $\Omega$, i.e $A=\{v \in \Omega: v g=g v, g \in G\}$. The orbit graph $\Gamma_{G}^{\Omega}$ consists of two sets, namely vertices and edges denoted by $V\left(\Gamma_{G}^{\Omega}\right)$ and $E\left(\Gamma_{G}^{\Omega}\right)$, respectively. The vertices of $\Gamma_{G}^{\Omega}$ are non central elements in $\Omega$ but not in $A$, that is $V\left(\Gamma_{G}^{\Omega}\right)=\Omega-A$, while the number of edges are $\left|E\left(\Gamma_{G}^{\Omega}\right)\right|=\sum_{i=1}^{\left|V\left(\Gamma_{G}^{\Omega}\right)\right|}\left(\begin{array}{c}v_{i} \\ 2\end{array}\right)$, where $v$ is the size of orbit under group action of $G$ on $\Omega$. Two vertices $v_{1}, v_{2}$ are adjacent in $\Gamma_{G}^{\Omega}$ if one of the following conditions is satisfied. 
1. If there exists $g \in G$ such that $g v_{1}=v_{2}$,

2. If the vertices of $\Gamma_{G}^{\Omega}$ are conjugate that is, $v_{1}=g^{v_{2}}$.

The orbit graph is also found for dihedral groups [5], solvable groups [6], symmetric groups and alternating groups [7].

As a connection between algebraic theory and graph theory, some works related to the probability that a group element fixes a set is stated as follows:

The commutativity degree is the probability that group elements commute. In 2014, El-sanfaz et al. [8] extended the work on the commutativity degree by defining the probability that a group element fixes a set. The following is the definition of the probability that a group element fixes a set.

Definition 2. [8] Let $G$ be a group. Let $S$ be a set of elements of $G$ of size two in the form of $(a, b)$ where $a$ and $b$ commute and $|a|=|b|=2$. Let $\Omega$ be the set of all subsets of commuting elements of $G$ of size two and $G$ acts on $\Omega$. Then the probability that an element of a group fixes a set is:

$$
P_{G}(\Omega)=\frac{\mid\{(g, \omega) \in G \times \Omega \mid g \omega=\omega \text { for } g \in G \text { and } \omega \in \Omega\} \mid}{|\Omega||G|} .
$$

The following theorem provides the probability that an element of a group fixes a set $\Omega$ in terms of the number orbits of $\Omega$ under group action of $G$ on $\Omega$.

Theorem 3. [8] Let $G$ be a finite group. Let $S$ be a set of elements of $G$ of size two in the form of $(a, b)$ where $a$ and $b$ commute and $|a|=|b|=2$. Let $\Omega$ be the set of all subsets of commuting elements of $G$ of size two and $G$ acts on $\Omega$. Then the probability that an element of a group fixes a set is given by:

$$
P_{G}(\Omega)=\frac{K(\Omega)}{|\Omega|},
$$

where $K(\Omega)$ is the number of orbits of $\Omega$ in $G$.

The probability can be computed under some group actions on a set. Elsanfaz et al. [9] used the conjugate action to find the probability that a group element fixes a set for metacyclic 2-groups of negative type, metacyclic 2-groups of positive type [10], semi-dihedral groups and quasi-dihedral groups [11].

Recently, El-sanfaz et al. [12] found the probability that an element of metacyclic 2-group of negative type fixes a set under regular action. The following are some of their results that are needed and used in this paper.

Theorem 4. [12] Let $G$ be a group of type (1), $G \cong\left\langle a, b: a^{2^{\alpha}}=b^{2^{\beta}}=\right.$ $\left.1,[b, a]=a^{2^{\alpha-\gamma}}\right\rangle$, where $\alpha, \beta, \gamma \in \mathbb{N}, \alpha \geq 2 \gamma$ and $\beta \geq \gamma \geq 1$. If $G$ acts regularly 
on $\Omega$, then

$$
P_{G}(\Omega)=\left\{\begin{array}{l}
\frac{5}{6}, \text { if } \beta=\gamma+1 \text { and } \alpha=2, \\
1, \text { otherwise. }
\end{array}\right.
$$

Remark 5. [12] The probability of the group of type (2) cannot be computed since there is only one element in $G$ that has of order two.

Theorem 6. [12] Let $G$ be a group of type (3), $G \cong\left\langle a, b: a^{2^{\alpha}}=1, b^{2}=\right.$ $\left.a^{2^{\alpha-1}},[b, a]=a^{-2}\right\rangle, \alpha \geq 3$. If $G$ acts regularly on $\Omega$, then $P_{G}(\Omega)=\frac{5}{6}$.

Theorem 7. [12] Let $G$ be group of type (4), $G \cong\left\langle a, b: a^{2^{\alpha}}=b^{2}=\right.$ $\left.1,[b, a]=a^{-2}\right\rangle, \alpha \geq 3$. If $G$ acts regularly on $\Omega$, then $P_{G}(\Omega)=\frac{5}{|\Omega|}$.

Theorem 8. [12] Let $G$ be a group of type (5), $G \cong\left\langle a, b: a^{2^{\alpha}}=b^{2}=\right.$ $\left.1,[b, a]=a^{2^{\alpha-1}-2}\right\rangle, \alpha \geq 3$. If $G$ acts regularly on $\Omega$, then

$$
P_{G}(\Omega)=\left\{\begin{array}{l}
\frac{2^{\alpha}+1}{|\Omega|}, \text { if } \alpha=3 \\
\frac{5}{|\Omega|}, \text { otherwise }
\end{array}\right.
$$

Theorem 9. [12] Let $G$ be a group of type (6), $G \cong\left\langle a, b: a^{2^{\alpha}}=b^{2^{\beta}}=\right.$ $\left.1,[b, a]=a^{-2}\right\rangle, \alpha \geq 3, \beta>1$. If $G$ acts regularly on $\Omega$, then $P_{G}(\Omega)=1$.

Remark 10. [12] The probability that a group element fixes a set under regular action of groups of types (7)-(10) are the same as in Theorem 9.

The following theorem illustrates the case when the probability of a group element fixes a set is equal to one.

Theorem 11. [12] Let $G$ be a finite non-abelian group. Let $S$ be a set of elements of $G$ of size two in the form of $(a, b)$, where $a$ and $b$ commute and $|a|=|b|=2$. Let $\Omega$ be the set of all subsets of commuting elements of $G$ of size two. If $G$ acts on $\Omega$ by conjugation, then $P_{G}(\Omega)=1$ if and only if all commuting elements $a$ and $b$ are in the center of $G$.

The next theorem provides the key connection between the probability that a group element fixes a set and the orbit graph.

Theorem 12. [11] Let $G$ be a finite non-abelian group and let $\Omega$ be the set of all subsets of commuting elements of $G$ of size two. If $G$ acts on $\Omega$ by conjugation and $P_{G}(\Omega)=1$, then $\Gamma_{G}^{\Omega}$ is a null graph.

The following result is used in this paper to show the case that the number of elements of $\Omega$ is equal to the number of orbits under group action on the set.

Theorem 13. [9] Let $G$ be a group of type (1), $G \cong\left\langle a, b: a^{2^{\alpha}}=b^{2^{\beta}}=\right.$ $\left.1,[b, a]=a^{2^{\alpha-\gamma}}\right\rangle$, where $\alpha, \beta, \gamma \in \mathbb{N}, \alpha \geq 2 \gamma$ and $\beta \geq \gamma \geq 1$. If $G$ acts on $\Omega$ by 
conjugation, then

$$
P_{G}(\Omega)=\left\{\begin{array}{l}
\frac{4}{|\Omega|}, \text { if } \beta=\gamma+1 \text { and } \alpha=2, \\
1, \text { if } \beta>1, \gamma=1 \text { and } \alpha \geq 3, \\
\frac{2}{|\Omega|}, \text { if } \beta>1, \gamma+1 \text { and } \alpha>3 .
\end{array}\right.
$$

Recently, El-sanfaz and Sarmin [13] found the probability that a group element fixes a set of metacyclic 2-groups of positive type. The results in [13] are then applied to generalized conjugacy class graph which is defined as a graph whose vertices are non-central orbits under group action on a set in which two vertices are adjacent if their cardinalities are not coprime.

\section{Main Results}

In this section, we present our results on the orbit graph of metacyclic 2-groups of negative type of nilpotency class two and class at least three, respectively. We start with the orbit graph of metacyclic group of the first type.

Theorem 14. Let $G$ be a group of type (1), $G \cong\left\langle a, b: a^{2^{\alpha}}=b^{2^{\beta}}=\right.$ $\left.1,[b, a]=a^{2^{\alpha-\gamma}}\right\rangle$, where $\alpha, \beta, \gamma \in \mathbb{N}, \alpha \geq 2 \gamma$ and $\beta \geq \gamma \geq 1$. If $G$ acts regularly on $\Omega$, then

$$
\Gamma_{G}^{\Omega}=\left\{\begin{array}{l}
K_{2}, \text { if } \beta=\gamma=1 \text { and } \alpha=2, \\
K_{0}, \text { if } \beta>1, \gamma=1 \text { and } \alpha \geq 3, \\
K_{e}, \text { if } \beta>1, \gamma>1 \text { and } \alpha>3 .
\end{array}\right.
$$

Proof. Let $\beta=\gamma=1$ and $\alpha=2$. Using Definition 1, the number of vertices in $\Gamma_{G}^{\Omega}$ is six. Based on the vertices adjacency of $\Gamma_{G}^{\Omega}$, the vertices which are in the form of $\left(a^{i} b, a^{i+2^{\alpha-1}} b\right), 0 \leq i \leq 2^{\alpha}$ are adjacent to each other, thus we have a complete graph of $K_{2}$, and we have four isolated vertices are in the form of $\left(a^{i} b, a^{2^{\alpha-2}}\right), 0 \leq i \leq 2^{\alpha}$. Hence, $\Gamma_{G}^{\Omega}$ consists one complete component of $K_{2}$. Next, when $\beta>1, \gamma=1$ and $\alpha \geq 3$, Based on Theorem 13, $P_{G}(\Omega)=1$. Using Theorem 12, we have $|\Omega|=|A|=K(\Omega)$ hence $\left|V\left(\Gamma_{G}^{\Omega}\right)\right|=0$. Therefore, $\Gamma_{G}^{\Omega}$ is null. Now, when $\beta>1, \gamma>1$ and $\alpha>3$, the number of elements of $\Omega$ is 3 , thus the number of vertices in $\Gamma_{G}^{\Omega}$ is 3 . Based on vertices adjacency of $\Gamma_{G}^{\Omega}$, thus there are 3 isolated vertices, namely $\left(a^{2^{\alpha-1}}, a^{2^{\alpha-1}} b^{2^{\beta-1}}\right),\left(b^{2^{\beta-1}}, a^{2^{\alpha-1}} b^{2^{\beta-1}}\right)$ and $\left(a^{2^{\alpha-1}}, b^{2^{\beta-1}}\right)$. Thus, graph in this case is empty. The proof is complete.

Remark 15. Based on Remark 5, the orbit graph of metacyclic 2-group of type (2) cannot be determined. 
Next, the orbit graph of metacyclic 2-groups of negative type of nilpotency class at least three is found, starting with the group of type (3).

Theorem 16. Let $G$ be a group of type (3), $G \cong\left\langle a, b: a^{2^{\alpha}}=1, b^{2}=\right.$ $\left.a^{2^{\alpha-1}},[b, a]=a^{-2}\right\rangle, \alpha \geq 3$. If $G$ acts regularly on $\Omega$, then $\Gamma_{G}^{\Omega}=K_{2}$.

Proof. Based on Theorem 6, the number of elements of $\Omega$ is six. Using Definition 1 , the number of vertices in $\Gamma_{G}^{\Omega}$ is six. According to vertices adjacency, the vertices that are in the form of $\left(a^{2^{\alpha-3}} i b, a^{2^{\alpha-3} i+2^{\alpha-1}}\right), 0 \leq i \leq 2^{\alpha}$ where $i$ is odd are adjacent to each other. The graph $\Gamma_{G}^{\Omega}$ also consists of four isolated vertices, namely $\left(a^{2^{\alpha-1}}, a^{2^{\alpha-3}} i b\right), 0 \leq i \leq 2^{\alpha}$. Therefore, the orbit graph of this group consists one complete component of $K_{2}$, as desired.

Based on Theorem 16, the following corollary is concluded.

Corollary 17. Let $G$ be a group of type (3), $G \cong\left\langle a, b: a^{2^{\alpha}}=1, b^{2}=\right.$ $\left.a^{2^{\alpha-1}},[b, a]=a^{-2}\right\rangle, \alpha \geq 3$. If $G$ acts regularly on $\Omega$ and $\Gamma_{G}^{\Omega}=K_{2}$, then $\chi\left(\Gamma_{G}^{\Omega}\right)=\omega\left(\Gamma_{G}^{\Omega}\right)=2$.

Proof. Since the graph consists of one complete component of $K_{2}$, thus the minimum number of coloring vertices is equal to two and the maximum number of complete subgraphs is also equal to two, as required.

Theorem 18. Let $G$ be group of type (4), $G \cong\left\langle a, b: a^{2^{\alpha}}=b^{2}=1,[b, a]=\right.$ $\left.a^{-2}\right\rangle, \alpha \geq 3$. If $G$ acts regularly on $\Omega$, then $\Gamma_{G}^{\Omega}=K_{2}$.

Proof. The proof is similar to the proof of Theorem 16.

Theorem 19. Let $G$ be a group of type (5), $G \cong\left\langle a, b: a^{2^{\alpha}}=b^{2}=\right.$ $\left.1,[b, a]=a^{2^{\alpha-1}-2}\right\rangle, \alpha \geq 3$. If $G$ acts regularly on $\Omega$, then

$$
\Gamma_{G}^{\Omega}=\left\{\begin{array}{l}
K_{4}, \text { if } \alpha=3 \\
K_{2}, \text { otherwise }
\end{array}\right.
$$

Proof. According to Theorem 8 and when $\alpha=3$, we have $G \cong D_{16}$. Using Definition 1, the number of vertices in $\Gamma_{G}^{\Omega}$ is 12 . Based on the vertices adjacency of the orbit graph, the vertices which are in the form of $\left(a^{i} b, a^{i+2^{\alpha-1}} b\right), 0 \leq i \leq$ $2^{\alpha}$ are adjacent to each other, hence we have a complete component of $K_{4}$ and eight isolated vertices, namely $\left(a^{i} b, a^{2^{\alpha-1}}\right), 0 \leq i \leq 2^{\alpha}$. Therefore, $\Gamma_{G}^{\Omega}$ consists of one complete component of $K_{4}$ and eight isolated vertices. Next, when $\alpha>3$, the proof is similar to the proof of Theorem 16.

According to Theorem 19, the following result can be concluded. 
Corollary 20. Let $G$ be a group of type (5), $G \cong\left\langle a, b: a^{2^{\alpha}}=b^{2}=\right.$ $\left.1,[b, a]=a^{2^{\alpha-1}-2}\right\rangle, \alpha \geq 3$. If $G$ acts regularly on $\Omega$ and $\Gamma_{G}^{\Omega}=K_{4}$, if $\alpha=3$, then $\chi\left(\Gamma_{G}^{\Omega}\right)=\omega\left(\Gamma_{G}^{\Omega}\right)=4$.

Proof. The proof is clear since there is one complete graph of $K_{4}$ when $\alpha=3$.

Next, the orbit graph of group of type (6) is determined.

Theorem 21. Let $G$ be a group of type (6), $G \cong\left\langle a, b: a^{2^{\alpha}}=b^{2^{\beta}}=\right.$ $\left.1,[b, a]=a^{-2}\right\rangle, \alpha \geq 3, \beta>1$. If $G$ acts regularly on $\Omega$, then

$$
\Gamma_{G}^{\Omega}=\left\{\begin{array}{l}
K_{0}, \text { if } \alpha=3, \\
K_{e}, \text { otherwise }
\end{array}\right.
$$

Proof. If $\alpha=3$, the proof is similar to the second part of the proof of Theorem 14. Next if $\alpha>3$, and based on Theorem 9, the number of elements of $\Omega$ is three. Therefore the number of vertices in $\Gamma_{G}^{\Omega}$ is three i.e. $\left|V\left(\Gamma_{G}^{\Omega}\right)\right|=3$. According to Definition 1, two vertices in $\Gamma_{G}^{\Omega}$ are adjacent to each other if there exists $g \in G$ and $\omega_{1}, \omega_{2} \in \Omega$ such that $g \omega_{1}=\omega_{2}$. Since $P_{G}(\Omega)=1$, there are three isolated vertices that are in the form of $\left(a^{2^{\alpha-1}}, b^{2^{\beta-1}}\right),\left(a^{2^{\alpha-1}}, a^{2^{\alpha-1}} b^{2^{\beta-1}}\right)$ and $\left(b^{2^{\beta-1}}, a^{2^{\alpha-1}} b^{2^{\beta-1}}\right)$. Thus the graph in this case is empty.

Theorem 22. Let $G$ be a group of type (7), $G \cong\left\langle a, b: a^{2^{\alpha}}=b^{2^{\beta}}=\right.$ $\left.1,[b, a]=a^{2^{\alpha-1}-2}\right\rangle$, where $\alpha \geq 3, \beta>1$. If $G$ acts regularly on $\Omega$, then

$$
\Gamma_{G}^{\Omega}=\left\{\begin{array}{l}
K_{0}, \text { if } \alpha=3, \\
K_{e}, \text { otherwise. }
\end{array}\right.
$$

Proof. The proof is similar to the proof of Theorem 21.

Theorem 23. Let $G$ be a group of type (8), $G \cong\left\langle a, b: a^{2^{\alpha}}=1, b^{2^{\beta}}=\right.$ $\left.a^{2^{\alpha-1}},[b, a]=a^{2^{\alpha-\gamma}-2}\right\rangle$, where $\alpha-\gamma>1, \beta>\gamma>1$. If $G$ acts regularly on $\Omega$, then $\Gamma_{G}^{\Omega}=K_{e}$.

Proof. The proof is similar to the second part of Theorem 21.

Theorem 24. Let $G$ be a group of type (9), $G \cong\left\langle a, b: a^{2^{\alpha}}=b^{2^{\beta}}=\right.$ $\left.1,[b, a]=a^{2^{\alpha-\gamma}-2}\right\rangle$, where $\alpha-\gamma>1, \beta \geq \gamma>1$. If $G$ acts regularly on $\Omega$, then

$$
\Gamma_{G}^{\Omega}=\left\{\begin{array}{l}
K_{0}, \text { if } \alpha=3, \\
K_{e}, \text { otherwise. }
\end{array}\right.
$$


Proof. The proof is similar to the proof of Theorem 21.

Theorem 25. Let $G$ be a group of type (10), $G \cong\left\langle a, b: a^{2^{\alpha}}=1, b^{2^{\beta}}=\right.$ $\left.a^{2^{\alpha-1}},[b, a]=a^{-2}\right\rangle$, where $\alpha \geq 3, \beta>1$. If $G$ acts regularly on $\Omega$, then $\Gamma_{G}^{\Omega}=K_{e}$.

Proof. The proof is similar to the second part of Theorem 21.

\section{Conclusion}

In this paper, the orbit graph of metacyclic 2-groups of negative type of nilpotency class two and class at least three is determined. Besides, some graph properties are provided which include the chromatic number, the clique number, the independence number and the dominating number.

\section{Acknowledgments}

The first author would like to acknowledge the Ministry of Higher Education in Libya for his PhD scholarship. The authors would also like to acknowledge Ministry of Education (MOE) Malaysia and Universiti Teknologi Malaysia for the financial funding through the Research University Grant (GUP) Vote No $08 \mathrm{H} 07$.

\section{References}

[1] J. Bondy, G. Murty, Graph Theory with Application, North Holand, Boston New York, 5th (1982).

[2] C. Godsil, G. Royle, Algebraic Graph Theory, Springer, Boston New York, 5th (2001).

[3] S.M.S. Omer, Extension of the Commutativity Degree of Some Finite Groups and Their Related Graphs, PhD Thesis, (2014).

[4] J.R. Beuerle, An elementary Classification of Finite Metacyclic p-groups of Class at Least Three, Algebra Colloq., 12 (2005), 553-562.

[5] S.M.S. Omer, N.H. Sarmin and A. Erfanian, The Orbit Graph of Finite Non-abelian Groups, International Journal of Pure and Applied Mathematics., 102(4) (2015), 747755 .

[6] S.M.S. Omer, N.H. Sarmin and A. Erfanian, The Orbit Graph for Some Finite Solvable Groups, AIP Conf. Proc., 1602, (2014), 863.

[7] S.M.S. Omer, N.H. Sarmin and A. Erfanian, The Probability that an Element of a Symmetric Group Fixes a Set and Its Application in Graph Theory, World Applied Sciences Journal., 27(12) (2013), 1637-1642. 
[8] M.A. El-sanfaz, N.H. Sarmin and S.M.S. Omer, The Probability That an Element of the Dihedral Groups Fixes a Set, International Journal of Applied Mathematics and Statistics, 51(1) (2014), 1-6.

[9] M.A. El-sanfaz, N.H. Sarmin and S.M.S. Omer, The Probability that an Element of Metacyclic 2-Groups Fixes a Set, World Applied Sciences Journal., 32(3) (2014), 459464.

[10] M.A. El-sanfaz, N.H. Sarmin and S.M.S. Omer, The Probability that an Element of Metacyclic 2-Groups of Positive Type Fixes a Set, Jurnal Teknologi., 71(1) (2014), 710.

[11] M.A. El-sanfaz, N.H. Sarmin and S.M.S. Omer, On the Probability that a Group Element Fixes a Set and its Generalized Conjugacy Class Graph, International Journal of Mathematical Analysis., 9(4) (2015), 161-167.

[12] M.A. El-sanfaz, N.H. Sarmin, The Probability That a Group Element Fixes a Set Under Regular Action for Metacyclic 2-Groups of Negative Type, International Journal of Applied Engineering Research., In press.

[13] M.A. El-sanfaz, N.H. Sarmin, On the Probability That an Element of Metacyclic 2-Group of Positive Type Fixes a Set and Its Generalized Conjugacy Class Graph, Global Journal of Pure and Applied Mathematics., 11(2) (2015), 899-908. 